\title{
Thermal transport in porous Si nanowires from approach-to-equilibrium molecular dynamics calculations
}

\author{
Xavier Cartoixà, ${ }^{1}$ Riccardo Dettori, ${ }^{2}$ Claudio Melis, ${ }^{2,3}$ Luciano Colombo, ${ }^{2,3,4,5}$ \\ and Riccardo Rurali ${ }^{5, a)}$ \\ ${ }^{1}$ Departament d'Enginyeria Electrònica, Universitat Autònoma de Barcelona, 08193 Bellaterra, Barcelona, \\ Spain \\ ${ }^{2}$ Dipartimento di Fisica, Università di Cagliari, Cittadella Universitaria, I-09042 Monserrato (Ca), Italy \\ ${ }^{3}$ Istituto Officina dei Materiali, CNR-IOM SLACS Cagliari, Cittadella Universitaria, I-09042 Monserrato \\ (Ca), Italy \\ ${ }^{4}$ Catalan Institute of Nanoscience and Nanotechnology (ICN2), CSIC and The Barcelona Institute of Science \\ and Technology, Campus UAB, 08193 Bellaterra, Barcelona, Spain \\ ${ }^{5}$ Institut de Ciència de Materials de Barcelona (ICMAB-CSIC), Campus de Bellaterra, 08193 Bellaterra, \\ Barcelona, Spain
}

(Received 26 March 2016; accepted 19 June 2016; published online 7 July 2016)

\begin{abstract}
We study thermal transport in porous Si nanowires (SiNWs) by means of approach-to-equilibrium molecular dynamics simulations. We show that the presence of pores greatly reduces the thermal conductivity, $\kappa$, of the SiNWs as long mean free path phonons are suppressed. We address explicitly the dependence of $\kappa$ on different features of the pore topology — such as the porosity and the pore diameter-and on the nanowire (NW) geometry-diameter and length. We use the results of the molecular dynamics calculations to tune an effective model, which is capable of capturing the dependence of $\kappa$ on porosity and NW diameter. The model illustrates the failure of Matthiessen's rule to describe the coupling between boundary and pore scattering, which we account for by the inclusion of an additional empirical term. Published by AIP Publishing.
\end{abstract}

[http://dx.doi.org/10.1063/1.4955038]

Thermoelectric materials can convert a thermal gradient into electricity. They represent one of the most appealing clean energy sources, as they allow transforming heatnormally regarded as a source of loss-into useful energy that can be easily harvested and stored. Unfortunately, the low efficiency of these materials has prevented their widespread use, confining them to niche applications. The thermoelectric efficiency is normally expressed by the dimensionless figure of merit $Z T=S^{2} T \sigma / \kappa$, where $S$ is the Seebeck coefficient, $T$ is the temperature, and $\sigma$ and $\kappa$ are electrical conductivity and thermal conductivity, respectively. Therefore, the design of new materials whose lattice thermal conductivity is more largely affected than the electrical conductivity by some additional structural features is one of the most active research lines in thermoelectricity.

Semiconducting nanowires $(\mathrm{NWs})^{1}$ and nanoporous materials $^{2,3}$ are both good examples of such an approach. A suitable distribution of voids, whose typical size is smaller than the mean-free-path of heat carriers, but still larger than the charge carrier one, results in strong scattering of the former, with negligible effects on the electrical conductivity. ${ }^{4,5}$ In nanowires, on the other hand, heat carriers are scattered at the wire surface, while charge carriers are comparatively much less affected. Accordingly, this effect is stronger in thin nanowires, with a larger surface-to-volume ratio, ${ }^{6}$ and in the presence of rough surfaces ${ }^{7,8}$ or other kinds of modulations. ${ }^{9}$

Naturally, the possibility to bring these two features together is very appealing. From a few years on, single crystalline porous silicon nanowires (pSiNWs) can be fabricated ${ }^{10}$ and have been proposed for different applications, ranging

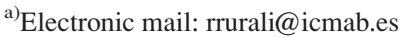

from lithium ion batteries ${ }^{11}$ to solar cells. ${ }^{12}$ However, their use as thermoelectric materials is to date unexplored. The goal of this paper is filling this gap and quantifying the reduction of the thermal conductivity in pSiNWs, as well as investigating its dependence on some specific features of the pore distribution - the overall porosity, the pore mean diameter-and of the nanowires-the diameter and the length. To this purpose, we first present a simple model describing the leading effects of all these factors, followed by the comparison to molecular dynamics simulations.

A first attempt at capturing both the effects of nanosized pores and the finite NW diameter can be easily constructed by adding a boundary scattering term ${ }^{13,14}$ to the expression for the mean free path through Matthiessen's rule, extending the modification to the Eucken model that we had previously proposed for bulk porous silicon $(\mathrm{pSi})^{5}$

$$
\begin{aligned}
\Lambda^{-1} & =\Lambda_{\text {anhamonicity }}^{-1}+\Lambda_{\text {porosity }}^{-1}+\Lambda_{\text {boundaries }}^{-1} \\
& =\Lambda_{\text {bulk }}^{-1}+\left(\frac{1}{\Lambda_{\text {bulk }}} \frac{\varphi}{2}+\frac{3 \varphi}{2 d_{p}}\right)+\frac{1-p}{1+p} \frac{\alpha}{d_{\mathrm{NW}}},
\end{aligned}
$$

where $\Lambda$ is the mean free path of heat carriers accounting for all scattering mechanisms, $\Lambda_{i}$ is the mean free path due to the $i$-th scattering mechanism $(i \in\{$ anharmonicity, porosity, boundaries $\}), d_{p}$ is the pore diameter, $\Lambda_{\text {bulk }}$ is the mean free path limited by phonon-phonon scattering in bulk material, $\varphi$ is the porosity $\varphi \equiv V_{\text {pores }} / V_{\text {system }}$, where $V$ is volume, $d_{\mathrm{NW}}$ is the NW diameter, and $\beta=\alpha(1-p) /(1+p)$ accounts for the shape of the NW cross section $[\alpha=1(\alpha=1.12)$ for circular (square) cross section $]^{13}$ and the surface polish ( $p=1$ for perfectly reflecting surfaces and $p=0$ for ideally rough surfaces). 
(a)

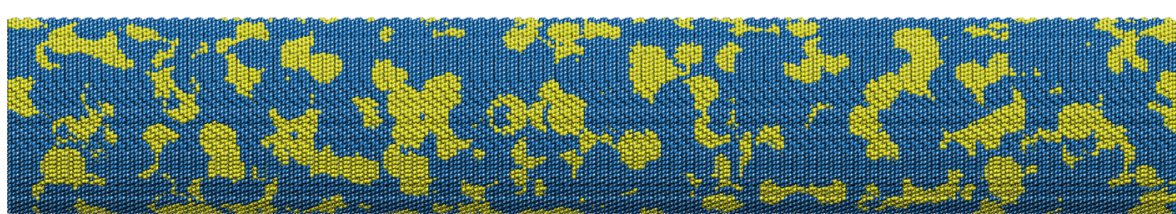

(b)

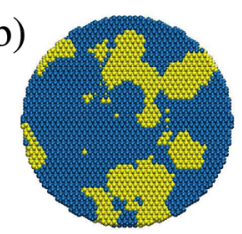

(c)

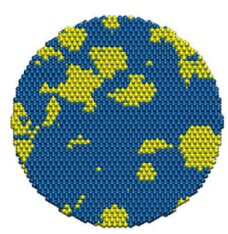

When Eq. (1) is entered into the kinetic expression for the thermal conductivity $\kappa=\frac{1}{3} \mathcal{C}_{\text {eff }} v_{g} \Lambda_{\text {eff }}$, and taking into account the modified value of the specific heat capacity $\mathcal{C}$ due to the presence of pores $\mathcal{C}_{\text {eff }}=(1-\varphi) \mathcal{C}_{\text {bulk }}$, we arrive at the model expression

$$
\frac{\kappa}{\kappa_{\text {bulk }}}=\frac{1-\varphi}{1+\frac{\varphi}{2}+\frac{3 \varphi}{2 d_{p}} \Lambda_{\text {bulk }}+\frac{\beta}{d_{\mathrm{NW}}} \Lambda_{\text {bulk }}} .
$$

It is important to remark that the model given in Eq. (1) is addressed to the leading scattering mechanisms, i.e., it is by construction adopting the same treatment of the boundary scattering as in non-porous NWs, thus assuming the "gray" Matthiessen's rule ${ }^{15}$ for phonon mean free paths to be valid in the presence of anharmonic, pore, and boundary scattering. The failure of Eq. (2) to describe all situations we have studied [see Fig. 3(b)] prompts us to relax the assumption of the validity of Matthiessen's rule, especially in light of previous works where appreciable coupling between anharmonic and boundary scattering in thin films ${ }^{16}$ and anharmonic and surface roughness scattering in ultrascaled $\mathrm{SiNWs}^{17}$ was found.

So, in order to account for the anharmonic-boundary coupling, we choose a simple linear expression $\Lambda_{\text {bulk }}$ $=\Lambda_{\text {bulk }, 0}\left(1-\gamma_{1} \varphi\right)$, where $\Lambda_{\text {bulk }, 0}$ is now the bulk mean free path limited by phonon-phonon scattering in bulk material and $\gamma_{1}$ encodes the reduction of the average anharmonic mean free path due to the disturbance in the distribution function because of the presence of pores. Similarly, in order to account for the pore-boundary coupling, we set $\beta=\beta_{0}$ $-\gamma_{2} \varphi$, where $\gamma_{2}$ describes the change in surface polish as porosity increases, and we introduce an empirical expression to adjust the coupling between porosity and surface scattering

$$
\Lambda_{\varphi-\mathrm{d}_{\mathrm{NW}}}^{-1}=\gamma_{3} \varphi / d_{\mathrm{NW}}^{\alpha},
$$

via a parameter $\gamma_{3}$ and an exponent $\alpha$. When all terms are included, the final version of the model is

$$
\kappa=\frac{\kappa_{\text {bulk }}(1-\varphi)}{\frac{1+\varphi / 2}{1-\gamma_{1} \varphi}+\frac{3 \varphi}{2 d_{p}} \Lambda_{\text {bulk }, 0}+\frac{\beta_{0}-\gamma_{2} \varphi}{d_{\mathrm{NW}}} \Lambda_{\text {bulk }, 0}+\gamma_{3} \frac{\varphi}{d_{\mathrm{NW}}^{\alpha}} \Lambda_{\text {bulk }, 0}} .
$$

While there have been some experimental measurements of the thermal conductivity of pSiNWs,${ }^{18,19}$ its lack of
FIG. 1. (a) Side view of a pSiNW with 0.3 porosity. Dark blue spheres represent $\mathrm{Si}$ atoms, while the atoms that have been removed are displayed as light yellow spheres for visualization purposes. Panels (b), (c), and (d) show cross-section views at $z=L_{z} / 4, L_{z} / 2$, and $3 L_{z} / 4$, respectively. (d)

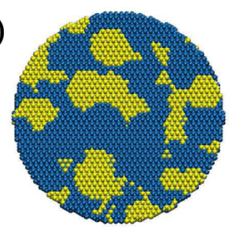

complete characterization in terms of NW diameter and porosity renders comparison to our model difficult. Thus, we compare the model in Eq. (2) to the thermal conductivity from approach-to-equilibrium molecular dynamics (AEMD) simulations. ${ }^{20}$ After equilibrating two roughly equal halves of the system to a hot and a cold temperature, $T_{H}$ and $T_{C}$, we study the transient to equilibrium during a microcanonical run. We then fit the time dependence of $\Delta T=T_{H}-T_{C}$ to the analytical solution of the heat equation, whose only fitting parameter is the thermal diffusivity $\bar{\kappa}$. We finally calculate the thermal conductivity as $\kappa=\bar{\kappa} \rho \mathcal{C}_{v}$, where $\rho$ is the density and $\mathcal{C}_{v}$ is the specific heat capacity. An extensive description of the method and additional details of the calculations can be found elsewhere. ${ }^{5,20}$

Our starting reference structure is a single crystalline SiNW oriented along the [111] crystallographic axis, with a diameter of $15 \mathrm{~nm}$ and a length of $90 \mathrm{~nm}$. As discussed in the text, we also consider diameters and lengths of up to 35 and $900 \mathrm{~nm}$, respectively, in some selected cases. Pores are created by randomly selecting atoms and drawing a sphere around them whose diameter is sampled from a Gaussian distribution with given mean value and variance, taken to be $1.5 \mathrm{~nm}$. Atoms inside the sphere are removed, creating a pore; the probability of creating a pore is adjusted to achieve a target porosity. Further iterations, where additional small pores are created or small portion of material restored, are then carried out until the desired porosity is obtained within a certain tolerance. As the AEMD method requires a uniform thermal conductivity along the transport direction, we apply the pore generation procedure above described within sequential regions of the sample, in order to guarantee a uniform porosity along the wire axis. We have considered porosities of 0.05 , $0.15,0.3$, and 0.5 , with average pore diameters-i.e., the mean value of the Gaussian distribution - of 2, 3, and $4 \mathrm{~nm}$. A typical structure is shown in Figure 1. Due to the random nature of pore formation, all the thermal conductivities discussed in this work have been obtained averaging over 3-4 independently generated configurations with the same target porosity and pore diameter. While this generation procedure is easily modifiable for a prescribed distribution of pore shapes and sizes, it still does not account for the oxidation at the pore surface. On the other hand, we observe an amorphization of the regions closest to the pore surfaces, which might mimic some characteristics of the native oxide, especially as the thermal conductivity of amorphous $\mathrm{Si}$ is similar to that of amorphous $\mathrm{SiO}_{2} \cdot{ }^{21}$

After generating the pores, we have minimized metastabilities in the structure by first performing a structural 
TABLE I. Fitted parameter values for Eqs. (2) and (4).

\begin{tabular}{lcc}
\hline \hline Parameter & Eq. (2) & Eq. (4) \\
\hline$\Lambda_{\text {bulk }, 0}(\mathrm{~nm})$ & 65.4 & 68.5 \\
$\gamma_{1}$ & $\ldots$ & 1.55 \\
$\beta_{0}$ & 0.24 & 0.22 \\
$\gamma_{2}$ & $\ldots$ & 75.5 \\
$\gamma_{3}\left(\mathrm{~nm}^{\alpha-1}\right)$ & $\ldots$ & 104.0 \\
$\alpha$ & $\ldots$ & 1.11 \\
\hline \hline
\end{tabular}

relaxation followed by a heating/cooling cycle up to $900 \mathrm{~K}$. This cycle was performed with periodic boundary conditions, so that unattached atoms or clusters eventually collided with the wire. After cooling back to $300 \mathrm{~K}$, further thermostatted thermalization proceeded for $200 \mathrm{ps}$, after which the thermostat was removed and the simulation continued for $400 \mathrm{ps}$ without appreciable temperature drift, indicating successful thermalization. At this point, a step-like temperature profile was set up by velocity rescaling for 200 ps for each half, after which the AEMD procedure was allowed to proceed for $1 \mathrm{~ns}$. We have used the environment dependent interatomic potential (EDIP) $)^{22}$ for the calculation of the energy and forces, and we have used a timestep of $2 \mathrm{fs}$ throughout all the simulation protocol. ${ }^{23}$

In what follows, we compare the AEMD results to our model expressions for the thermal conductivity $\kappa$ using the parameters in Table I. The parameters for Eq. (2) were obtained by a fit to the data in Figs. 2 and 3(a) only because inclusion of the points in Fig. 3(b) in the target dataset brought an insignificant improvement in Fig. 3(b), while significantly worsening the adjustment in Figs. 2 and 3(a). On the other hand, the parameters in Eq. (4) were chosen taking into account results in Fig. 3(b) in addition to Figs. 2 and 3(a).

The main result of our work is displayed in Figure 2, where we plot the thermal conductivity, $\kappa$, of a $15 \mathrm{~nm}$ diameter SiNW as a function of the porosity, achieved by randomly introducing pores of $3 \mathrm{~nm}$ diameter, comparing it to the fits through Eqs. (2) and (4). Notice that the value of

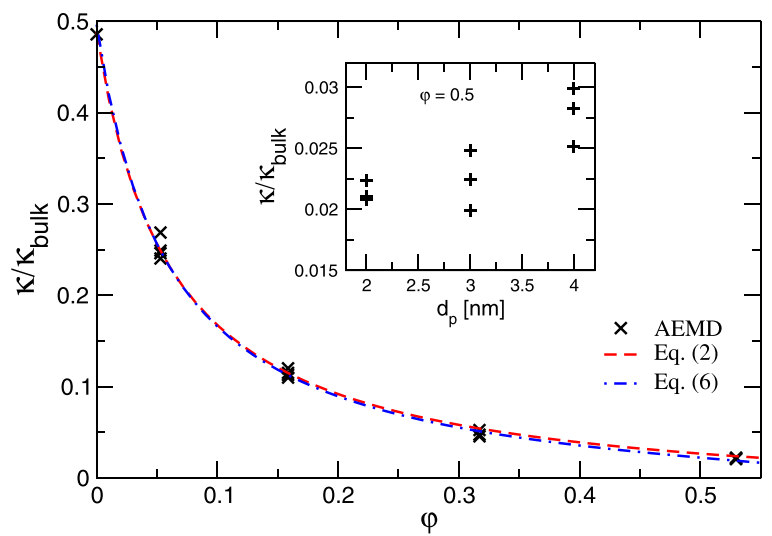

FIG. 2. Thermal conductivity as a function of the total porosity for pores of average diameter of $3 \mathrm{~nm}$ in a $15 \mathrm{~nm}$ diameter and $90 \mathrm{~nm}$ long SiNW. The estimated values of $\kappa$ are referred to the thermal conductivity of bulk Si, calculated in a computational cell of the same length and cross-section. The dashed red (dashed-dot blue) line corresponds to Eq. (2) [Eq. (4)] with the parameter values in Table I. The symbols in the main panel indicate the thermal conductivity of each sample. Inset: dependence of $\kappa$ on the pore diameter in the case of porosity $\sim 0.5$.

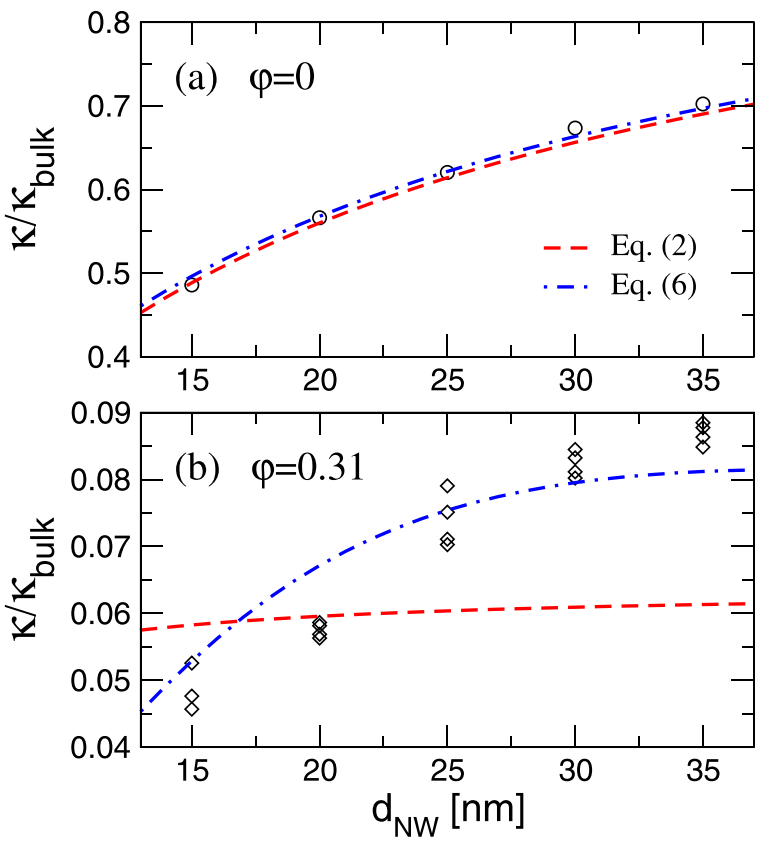

FIG. 3. Thermal conductivity as a function of the nanowire diameter, $d_{N W}$, for a pristine NW (left) and a porous NW with porosity $\sim 0.31$ and pores of average diameter of $3 \mathrm{~nm}$ (right). Values are referred to the thermal conductivity of bulk Si. Lines have the same meaning as that in Fig. 2.

$\Lambda_{\text {bulk,0 }}$ agrees well with the one fitted in our previous study of bulk pSi, where we obtained $40 \mathrm{~nm}<\Lambda_{\text {bulk }}<110 \mathrm{~nm}$. ${ }^{5}$ It is clear that the presence of nanovoids results in a very strong reduction of the thermal conductivity and a bare $5 \%$ of porosity is enough to decrease it approximately to half of the value of the pristine wire. The sample variation of $\kappa$ is a few percent. Notice that boundary scattering alone accounts for a reduction of a factor of two for the pristine $(\varphi=0)$ NW with respect to bulk ( $\kappa / \kappa_{\text {bulk }} \sim 0.5$ in Figure 2$)$, but the rather low porosity $\varphi=0.3$ results in an additional reduction of one order of magnitude $\left(\kappa / \kappa_{\text {bulk }} \sim 0.05\right)$. In the case of the highest porosity considered, $\varphi \sim 0.5$, we have studied the influence of the pore diameter (inset of Figure 2), comparing 2,3 , and $4 \mathrm{~nm}$ pores. A trend emerges, indicating that smaller pores result in slightly stronger reduction of $\kappa$. This effect was also observed in bulk pSi, and it is a manifestation that at the nanoscale the interface density plays a crucial role in the behavior of $\kappa^{5}$. Since Eq. (2) provides a good fit to the data, we see that the "gray" Matthiessen's rule for the mean free paths is valid when combining anharmonic and pore scattering mechanisms.

Figure 3 shows the NW diameter dependence of $\kappa$ for (a) a pristine $\mathrm{SiNW}$ and (b) a pSiNW with $\varphi=0.31$. The red (blue) lines correspond to the expression in Eq. (2) [Eq. (4)]. So, the predictive power of the model is clearly established for the case finite diameter NWs without pores [Figure 3(a)]. The predictions of the model (2) are much less satisfactory for the case of the NW with finite porosity [Figure 3(b)]; while the order of magnitude of $\kappa$ is correctly obtained, the model fails to reproduce the almost doubling of the conductivity as the NW diameter increases from $15 \mathrm{~nm}$ to $35 \mathrm{~nm}$. From this, the necessity to introduce terms in the model not conforming to Matthiessen's rule is evident. Physically, the failure of Matthiessen's rule in ultra-scaled SiNWs in the presence of surface roughness and anharmonic scattering has 

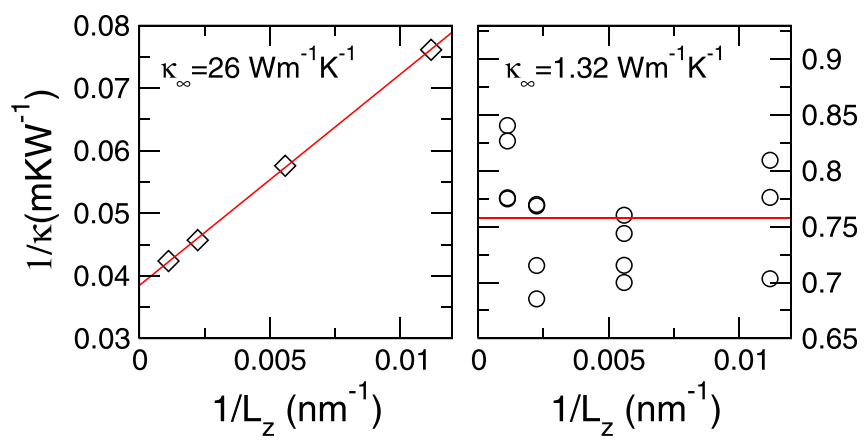

FIG. 4. Inverse of the thermal conductivity as a function of the inverse of the nanowire length along the transport direction, $L_{z}$, for a pristine NW (top) and a porous NW with porosity $\sim 0.3$ and pores of average diameter of $3 \mathrm{~nm}$ (bottom). A linear fit of the $\kappa^{-1}\left(L_{z}^{-1}\right)$ allows extrapolating the value of $\kappa_{\infty}$ for $L_{z}^{-1} \rightarrow 0$ for the pristine wire. In the case of the porous wire in absence of a clear $L_{z}$ dependence, we simply plot the mean value of the data points.

been attributed to anharmonic scattering allowing the decay of high-energy non-propagating (due to roughness) phonons into lower energy propagating states. ${ }^{17}$ It is conceivable that a similar mechanism might be at work in porous SiNWs, with the NW surface redistributing the number of propagons, diffusons, and locons ${ }^{24}$ expected to be present in these systems with impaired translational symmetry due to the presence of pores.

Finally, heat carriers with a wavelength larger than the simulation cell cannot be described, a well-known fact that results in an underestimation of the thermal conductivity. ${ }^{25,26}$ This is why, to be consistent, the reduction of all the values of $\kappa$ discussed so far has been calculated relatively to a cell of bulk $\mathrm{Si}$ of the same length. In general, the cell required to obtain a converged estimate of $\kappa$ in Si-based systems-where phonons with mean free paths of up to hundreds of $\mathrm{nm}$ can contribute to heat transport ${ }^{27}$-requires a very significant computational workload. The common method to circumvent this problem consists in extrapolating $\kappa^{-1}\left(L_{z}^{-1}\right)$ to $L_{z}^{-1} \rightarrow 0 .{ }^{28}$ We have followed this procedure for the pristine and a porous nanowire with porosity 0.3 . As seen in Figure 4, for the pristine nanowire, we obtain the wellknown linear dependence of $\kappa^{-1}\left(L_{z}^{-1}\right)$, which allows extrapolating a value of $\kappa_{\infty}=26.0 \mathrm{~W} \mathrm{~m}^{-1} \mathrm{~K}^{-1}$. The porous $\mathrm{NW}$, on the other hand, exhibits a very poor dependence on $L_{z}$, indicating that the smallest cell size considered already accounts for the mean free paths of all phonons contributing to the thermal conductivity. In this case, we simply plot the mean value of the calculated data points, because of the large uncertainty of the (small) fitted slope of $\kappa^{-1}\left(L_{z}^{-1}\right)$. Notice that this means that the reductions of $\kappa$ discussed thus far would be even larger, because we are underestimating the thermal conductivity of the non-porous wire: we obtained $\kappa_{L_{z}=90 \mathrm{~nm}}^{0.0} / \kappa_{L_{z}=90 \mathrm{~nm}}^{0.3} \sim 10$ for the $L_{z}=90 \mathrm{~nm}$ cell, while for long SiNWs, $\kappa_{\infty}^{0.0} / \kappa_{\infty}^{0.3} \sim 20$.

In summary, we have shown that the presence of pores, the pore interface separation becoming the new dominant length scale, suppresses the long mean free path phonon contribution to $\kappa$, thus causing a significant reduction of the already low thermal conductivity in SiNWs, which might open new avenues for improved thermoelectrics. We also provide a model expression extending the Eucken model that accurately predicts thermal conductivities in porous SiNWs. We find that the Matthiessen's rule applied to the phonon mean free paths does not hold due to the coupling effects between surface and pore scattering.

We acknowledge financial support by the Spanish MINECO under Grant Nos. FIS2012-37549-C05-02, MAT2013-40581-P, TEC2012-31330 (co-funded by the EU under the FEDER Program), and TEC2015-67462-C2-1-R (MINECO/FEDER) and through the Severo Ochoa Centres of Excellence Program under Grant Nos. SEV-2013-0295 and SEV-2015-0496; the Generalitat de Catalunya under Grant Nos. 2014 SGR 301 and 2014 SGR 384; the Regione Sardegna basic research Project No. CRP78744 "Energy Applications with Porous Silicon (ENAPSi)." One of us (R.D.) acknowledges Regione Sardegna for financial support under Project P.O.R. Sardegna F.S.E. 2007-2013 (Axis IV Human Resources, Objective 1.3, Line of Activity 1.3.1.). The calculations have been performed at the Barcelona Supercomputing Center (BSC-CNS) within the project "Nanoporous silicon for thermoelectric applications (FI-2015-1-0010).”

${ }^{1}$ W. Lu and C. M. Lieber, J. Phys. D: Appl. Phys. 39, R387 (2006); R. Rurali, Rev. Mod. Phys. 82, 427 (2010).

${ }^{2}$ L. T. Canham, Appl. Phys. Lett. 57, 1046 (1990).

${ }^{3}$ V. Lehmann and U. Gösele, Appl. Phys. Lett. 58, 856 (1991).

${ }^{4}$ J. Tang, H.-T. Wang, D. H. Lee, M. Fardy, Z. Huo, T. P. Russell, and P. Yang, Nano Lett. 10, 4279 (2010).

${ }^{5}$ R. Dettori, C. Melis, X. Cartoixà, R. Rurali, and L. Colombo, Phys. Rev. B 91, 054305 (2015).

${ }^{6}$ D. Li, Y. Wu, P. Kim, L. Shi, P. Yang, and A. Majumdar, Appl. Phys. Lett. 83, 2934 (2003).

${ }^{7}$ A. I. Boukai, Y. Bunimovich, J. Tahir-Kheli, J.-K. Yu, W. A. Goddard III, and J. R. Heath, Nature 451, 168 (2008).

${ }^{8}$ A. I. Hochbaum, R. Chen, R. D. Delgado, W. Liang, E. C. Garnett, M. Najarian, A. Majumdar, and P. Yang, Nature 451, 163 (2008).

${ }^{9}$ X. Zianni, V. Jean, K. Termentzidis, and D. Lacroix, Nanotechnology 25, 465402 (2014).

${ }^{10}$ Y. Qu, H. Zhou, and X. Duan, Nanoscale 3, 4060 (2011).

${ }^{11}$ M. Ge, J. Rong, X. Fang, and C. Zhou, Nano Lett. 12, 2318 (2012).

${ }^{12}$ A. Najar, J. Charrier, P. Pirasteh, and R. Sougrat, Opt. Express 20, 16861 (2012).

${ }^{13}$ H. Casimir, Physica 5, 495 (1938); J. M. Ziman, Electrons and Phonons (Oxford University Press, 1960), p. 463.

${ }^{14}$ J. Zou and A. Balandin, J. Appl. Phys. 89, 2932 (2001).

${ }^{15}$ T. Feng, B. Qiu, and X. Ruan, Phys. Rev. B 92, 235206 (2015).

${ }^{16}$ J. E. Turney, A. J. H. McGaughey, and C. H. Amon, J. Appl. Phys. 107, 024317 (2010).

${ }^{17}$ M. Luisier, Appl. Phys. Lett. 103, 113103 (2013).

${ }^{18}$ J. M. Weisse, A. M. Marconnet, D. R. Kim, P. M. Rao, M. A. Panzer, K. E. Goodson, and X. Zheng, Nanoscale Res. Lett. 7, 554 (2012).

${ }^{19}$ T. Zhang, S.-L. Wu, R.-T. Zheng, and G.-A. Cheng, Nanotechnology 24, 505718 (2013).

${ }^{20}$ C. Melis, R. Dettori, S. Vandermeulen, and L. Colombo, Eur. Phys. J. B 87, 96 (2014).

${ }^{21}$ J. M. Larkin and A. J. H. McGaughey, Phys. Rev. B 89, 144303 (2014).

${ }^{22}$ J. A. F. Justo, M. Z. Bazant, E. Kaxiras, V. V. Bulatov, and S. Yip, Phys. Rev. B 58, 2539 (1998).

${ }^{23}$ S. Plimpton, J. Comput. Phys. 117, 1 (1995).

${ }^{24}$ P. B. Allen, J. L. Feldman, J. Fabian, and F. Wooten, Philos. Mag. B 79, 1715 (1999).

${ }^{25}$ C. Oligschleger and J. C. Schön, Phys. Rev. B 59, 4125 (1999).

${ }^{26}$ P. K. Schelling, S. R. Phillpot, and P. Keblinski, Phys. Rev. B 65, 144306 (2002).

${ }^{27}$ K. T. Regner, D. P. Sellan, Z. Su, C. H. Amon, A. J. McGaughey, and J. A. Malen, Nat. Commun. 4, 1640 (2013).

${ }^{28}$ J. Michalski, Phys. Rev. B 45, 7054 (1992). 